\title{
Molecularly imprinted polymers and capillary electrophoresis for sensing phytoestrogens in milk
}

\author{
Jaroslava Bezdekova, ${ }^{1,2}$ Marcela Vlcnovska, ${ }^{1}$ Kristyna Zemankova, ${ }^{1}$ Romana Bacova, ${ }^{1,2}$ Martina Kolackova, ${ }^{1,2}$ \\ Tomas Lednicky, ${ }^{2}$ Jan Pribyl, ${ }^{3}$ Lukas Richtera, ${ }^{1,2}$ Lucie Vanickova, ${ }^{1,2}$ Vojtech Adam, ${ }^{1,2}$ \\ and Marketa Vaculovicova ${ }^{1,2 *}$ \\ ${ }^{1}$ Department of Chemistry and Biochemistry, Mendel University in Brno, Zemedelska 1, CZ-613 00 Brno, Czech Republic \\ ${ }^{2}$ Central European Institute of Technology, Brno University of Technology, Purkynova 123, CZ-612 00 Brno, Czech Republic \\ ${ }^{3}$ Central European Institute of Technology, Masaryk University, Kamenice 753/5 CZ-62500 Brno, Czech Republic
}

\begin{abstract}
Dairy cow feed contains, among other ingredients, soybeans, legumes, and clover, plants that are rich in phytoestrogens. Several publications have reported a positive influence of phytoestrogens on human health; however, several unfavorable effects have also been reported. In this work, a simple, selective, and ecofriendly method of phytoestrogen isolation based on the technique of noncovalent molecular imprinting was developed. Genistein was used as a template, and dopamine was chosen as a functional monomer. A layer of molecularly imprinted polymers was created in a microtitration well plate. The binding capability and selective properties of obtained molecularly imprinted polymers were investigated. The imprinted polymers exhibited higher binding affinity toward chosen phytoestrogen than did the nonimprinted polymers. A selectivity factor of 6.94 was calculated, confirming satisfactory selectivity of the polymeric layer. The applicability of the proposed sensing method was tested by isolation of genistein from a real sample of bovine milk and combined with micellar electrokinetic capillary chromatography with UV-visible detection
\end{abstract} Key words: biochanin A, genistein, dopamine, milk

\section{INTRODUCTION}

Phytoestrogens are secondary plant metabolites exhibiting estrogenic activity (Murkies et al., 1998). Their structure is similar to the hormone estradiol; therefore, these substances are also known as nonsteroidal estro-

Received July 31, 2019.

Accepted January 8, 2020.

*Corresponding author: marketa.ryvolova@seznam.cz gens (Bennetau-Pelissero et al., 2000; Chrzanowska et al., 2015b). It has been found that phytoestrogens are able to interact with estrogen receptors (Tham et al., 1998).

Several publications have reported a positive influence of phytoestrogens on human health. According to these studies, phytoestrogens have positive effects on the prevention of osteoporosis and cardiovascular disease and can influence menopause symptoms (Patisaul and Jefferson, 2010; Fu et al., 2014; González Cañete and Durán Agüero, 2014). Conversely, phytoestrogens are also endocrine-disrupting compounds and therefore can cause unfavorable effects such as disruptions in sexual behavior and brain sexual differentiation, changes in hormone levels, and increases in breast cancer risk (Xiao, 2008; Chrzanowska et al., 2015b; Socas-Rodríguez et al., 2018). Hence, monitoring phytoestrogen levels is important to ensure the high quality and safety of food.

Generally, phytoestrogens occur in complex matrixes and in very low concentrations; thus, their detection often demands effective sample preparation before any instrumental analysis (Chrzanowska et al., 2015b). Liquid-liquid or solid-phase extraction are the most commonly used techniques for phytoestrogen isolation (Franke et al., 1998; Zheng et al., 1999; Cao et al., 2009). However, these methods require a large amount of solvents, which is incompatible with an eco-friendly approach.

Molecular imprinting is a technique that permits the production of tailor-made binding sites specific to the imprinted molecule (template) in shape, size, and functional groups in cross-linked polymers (Chen et al., 2016). Molecularly imprinted polymers (MIP) are highly applicable in chemical sensors due to their high chemical and physical stability and their ability to form the selective recognition binding sites specific to a variety of molecule types ( $\mathrm{Li}$ et al., 2012). In addition, they can be produced in large quantities and can be 
reused (Mattiasson and Ye, 2015). In comparison, commonly used biorecognition elements (antibodies) are usually very expensive and not always available for the targeted analyte, and, in a number of cases, significant cross-reactivity and nonspecificity may occur.

In this work, the novel, effective, and eco-friendly method of phytoestrogen isolation was suggested based on the technique of molecular imprinting. A noncovalent imprinting approach was used due to its simplicity. Dopamine was used as a functional monomer because the polymerization occurs in the presence of oxygen under alkaline conditions (Jiang et al., 2011), forming a stable and eco-friendly polydopamine layer (Zhao et al., 2018).

Polydopamine synthesis is most often carried out by commercially available dopamine hydrochloride reacting with a base (typically Tris) under aqueous aerobic conditions (Dreyer et al., 2013). When a dopamine is dissolved in a basic aqueous solution, the oxygen spontaneously dissolved in water causes oxidation of dopamine to quinone, followed by an intramolecular Michael addition, leading to the creation of leucodopamine (Perrot et al., 2016; Ball, 2018). Additional oxidation and rearrangement lead to the formation of intermediates (dopamine-chrome, dihydroxylindole, and indolequinone). Intermediates can create covalent linkages between repeat units in the polymer backbone at the positions adjacent to the oxygen functional groups (hydroxyls or ketones, depending on their oxidation state) as well as their respective positions on the nitrogen heterocycle, or they can be bonded in a noncovalent manner (Dreyer et al., 2013).

Genistein was used as a template and model analyte because of its presence in animal and human food (Chrzanowska et al., 2015b). The binding experiments were carried out to investigate binding properties of MIP compared with nonimprinted polymers (NIP) used as a control. The selectivity of MIP was verified using biochanin A, a competitive analyte. Finally, the extraction of genistein from a real milk sample was investigated.

\section{MATERIALS AND METHODS}

\section{Materials}

Dopamine hydrochloride, Trizma base, SDS, genistein, biochanin A, and acetic acid were purchased from Sigma-Aldrich (St. Louis, MO) in ACS grade. Sodium tetraborate decahydrate was obtained from Thermo Fisher Scientific (Waltham, MA). Ethanol and methyl alcohol were purchased from Penta (Prague, Czech Republic).

\section{Preparation of MIP}

The MIP and NIP were prepared in a microtitration well plate. For MIP, $5 \mathrm{mg}$ of dopamine was dissolved in $1 \mathrm{~mL}$ of genistein solution (genistein was dissolved in $80 \%$ ethanol). For NIP, the same amount of dopamine was dissolved in $80 \%$ ethanol only. Then, $1 \mathrm{~mL}$ of 20 $\mathrm{m} M$ Tris buffer (pH 10.2) was added to both MIP and NIP. Then, $100 \mu \mathrm{L}$ of the resulting solution was polymerized for $24 \mathrm{~h}$ at $25^{\circ} \mathrm{C}$ in the well plate. As the reaction progressed, the clear and colorless solution gradually turned brown. After polymerization reaction, the template (genistein) was removed by washing with $50 \mu \mathrm{L}$ of 90\% methanol 3 times. Prepared MIP layers were used for isolation of genistein from sample [aqueous standard solution or real sample (milk)].

\section{MIP and NIP Layer Characterization}

The prepared polymeric layers were characterized by atomic force microscopy, Fourier-transformation infrared spectroscopy, and MALDI-TOF MS imaging. Details are given in Supplemental File S1 (https://doi .org/10.3168/jds.2019-17367).

\section{Adsorption Properties of Prepared MIP and NIP}

The adsorption properties of MIP and NIP selective for genistein were evaluated by binding, kinetic, and selectivity adsorption experiments. All experiments were repeated 3 times, and the values are shown as the average of 3 measurements. To investigate binding abilities, $50 \mu \mathrm{L}$ of genistein of various concentrations $(0.0075,0.015,0.03,0.06,0.125,0.25$, and $0.5 \mathrm{mg} / \mathrm{mL})$ was added to MIP or NIP. After $30 \mathrm{~min}$ of interaction at room temperature, the supernatant containing unbound genistein was removed and the surface was washed with $50 \mu \mathrm{L}$ of water. Subsequently, the bound genistein was released with $50 \mu \mathrm{L}$ of $90 \%$ methanol and measured using micellar electrokinetic capillary chromatography. The kinetic adsorption experiment was carried out as follows: $50 \mu \mathrm{L}$ of $0.125 \mathrm{mg} / \mathrm{mL}$ genistein dissolved in $80 \%$ ethanol was added to prepare the MIP or NIP layer. Genistein reacted with MIP or NIP for different time intervals (5-35 min). Subsequently, the unbound genistein was removed (the surface was washed with $50 \mu \mathrm{L}$ of water), and the bound genistein was released with $50 \mu \mathrm{L}$ of $90 \%$ methanol. Released genistein was measured using micellar electrokinetic capillary chromatography. In the selectivity adsorption experiments, biochanin A was chosen as a competitor. This molecule contains 1 additional methyl group com- 

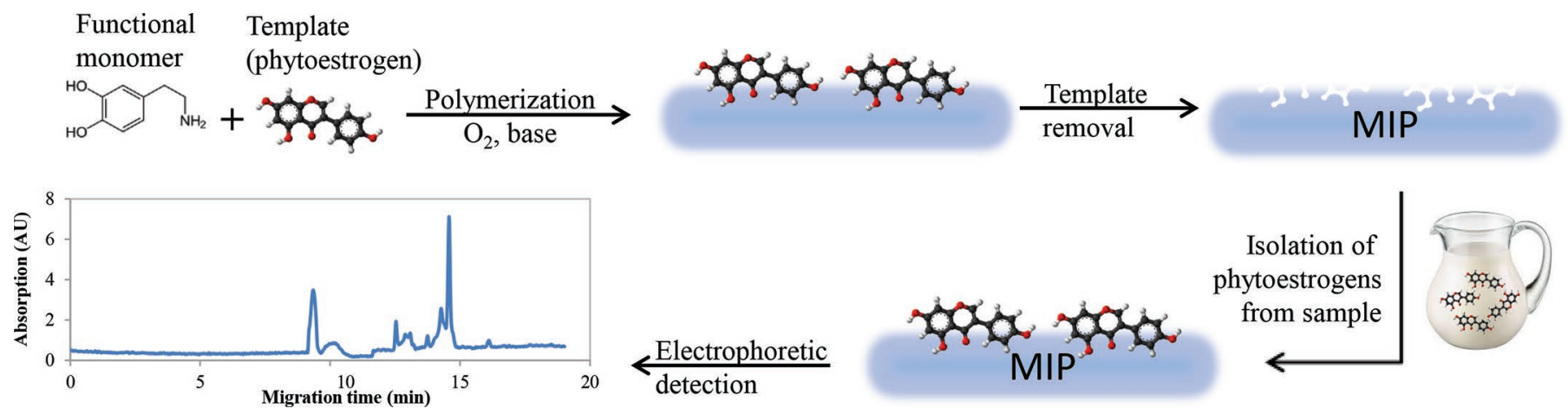

Figure 1. Scheme of the molecularly imprinted polymer (MIP) preparation process. AU = absorbance units.

pared with the genistein molecule. The experiment was carried out as follows: $50 \mu \mathrm{L}$ of $0.125 \mathrm{mg} / \mathrm{mL}$ biochanin A was added to the MIP or NIP layer (genistein was used as template). After $30 \mathrm{~min}$, the supernatant was removed (the surface was washed with $50 \mu \mathrm{L}$ of water). Bound biochanin A was released with $50 \mu \mathrm{L}$ of $90 \%$ methanol and measured using micellar electrokinetic capillary chromatography.

\section{Micellar Electrokinetic Capillary Chromatography}

Conditions for separation of genistein and biochanin A were as reported by Zhou et al. (2010). Briefly, quantification of phytoestrogens (genistein, biochanin A) was carried out using an Agilent 7100 Capillary Electrophoresis instrument (Agilent Technologies, Santa Clara, CA) with absorbance detection at 254 $\mathrm{nm}$. A fused silica capillary with an internal diameter of $75 \mu \mathrm{m}$, a total length of $64.5 \mathrm{~cm}$, and an effective length of $56 \mathrm{~cm}$ was used. The following traits were used: the sample was introduced hydrodynamically at $3.5 \mathrm{kPa}$ for $3 \mathrm{~s}$, and a separation voltage of 12 to 25 $\mathrm{kV}$ was optimized. A background electrolyte consisted of $30 \mathrm{~m} M$ borate and 10 to $40 \mathrm{~m} M$ SDS containing $5 \%$ (vol/vol) ethanol at $\mathrm{pH} 8$ to 10 . Before the analysis, the capillary was washed for $60 \mathrm{~s}$ using the background electrolyte.

\section{Isolation and Detection of Genistein from Bovine Milk Sample}

For verification of the applicability of the method, a bovine milk sample was used. The milk $(50 \mu \mathrm{L})$ was spiked with $2 \mu \mathrm{L}$ of genistein $(1 \mathrm{mg} / \mathrm{mL})$. Then, $50 \mu \mathrm{L}$ of crude bovine milk or spiked bovine milk was applied to the surface of MIP or NIP, and the interaction took $30 \mathrm{~min}$ at room temperature. Then, the supernatant was removed and isolated genistein was released with
$50 \mu \mathrm{L}$ of $90 \%$ methanol 3 times. Removed genistein was detected using micellar electrokinetic capillary chromatography. The final volume was $150 \mu \mathrm{L}$.

\section{RESULTS AND DISCUSSION}

\section{Approach Taken in this Work}

Figure 1 summarizes the overall scheme of the process. The polymer mixture (dopamine and genistein) underwent oxidative polymerization in alkaline solution on the selected surface (i.e., the bottom of the microtitration well plate), the template was removed, and prepared MIP was used for extraction of genistein from the milk sample. Finally, the extracted phytoestrogen was analyzed and detected using capillary electrophoresis with UV-visible detection.

The morphology of the prepared layer was controlled by atomic force microscopy. It was found that the layers in all studied cases were very heterogeneous (Supplemental Figure S1; https://doi.org/10.3168/jds .2019-17367), which is in agreement with previous studies. The chemical characterization was performed using Fourier-transformation infrared spectroscopy as shown in Supplemental Figure S2 (https://doi.org/10.3168/ jds.2019-17367). First, it was found that the polydopamine layer after polymerization with the template was providing the highest signals. After the template removal step, the intensities decreased dramatically, and after analyte binding, the signal increased. This trend is demonstrated in Supplemental Figure S2A (the individual steps are schematically shown in the inset). Moreover, the distinct signals were assigned to the structural features specific to dopamine and biochanin A (template and sample molecule). These are based on previous studies (Sekine et al., 2011; Capeletti and Zimnoch, 2016; Thakur et al., 2018) and are marked in Supplemental Figure S2B. 
Finally, the binding abilities were supported by MALDI-TOF MS imaging analysis. The results are shown in Supplemental Figure S3 (https://doi.org/10.3168/jds .2019-17367). Based on these results, it is clear that the NIP layer did not provide any signal of phytoestrogen as the polymerization was performed without presence of the template (Supplemental Figure S3A). On the contrary, after template removal the MIP layer exhibited low signal of the template trapped within the layer (Supplemental Figure S3B). Finally, the MIP layer after analyte rebinding is shown in Supplemental Figure S3C. The presented heat maps are specifically extracted for the biochanin A molecular mass.

\section{Determination of Phytoestrogens Using Micellar Electrokinetic Capillary Chromatography}

Phytoestrogens are neutral (uncharged) molecules; therefore, they are in the capillary electrophoresis and related techniques carried by the electroosmotic flow. Their separation under commonly used conditions is impossible. Hence, micellar electrokinetic capillary chromatography is required for separation. To enable the analysis, addition of micelle-forming compound such as SDS into the background electrolyte, exceeding the critical micellar concentration, is necessary. Formed micelles provide a negative charge and migrate against the electroosmotic flow. Neutral substances exhibit different affinity to such pseudostationary phase and are separated on the basis of different distribution coefficients (Terabe, 2009).

All phytoestrogens or their metabolites have in their structure at least 1 aromatic ring. This means that they are able to absorb UV light with a maximum wavelength in the range of 250 to $270 \mathrm{~nm}$ (Wang et al., 2002). Therefore, a wavelength of $254 \mathrm{~nm}$ seem to be an ideal choice.

To optimize the micellar electrokinetic capillary chromatographic separation, a mixture of genistein and biochanin $\mathrm{A}$ in a ratio of 1:1 was analyzed. The main parameters influencing the separation, such as separation voltage $(12,15,20$, and $25 \mathrm{kV})$, SDS concentration $(10,20,30$, and $40 \mathrm{mM})$, and $\mathrm{pH}(8,9,9.5$, and 10), were optimized. To evaluate the effect of these parameters, separation resolution was calculated (Figure 2). It was found that the voltage providing the separation with the highest resolution was $12 \mathrm{kV}$ and the optimal buffer $\mathrm{pH}$ was found to be 10 ; however, the

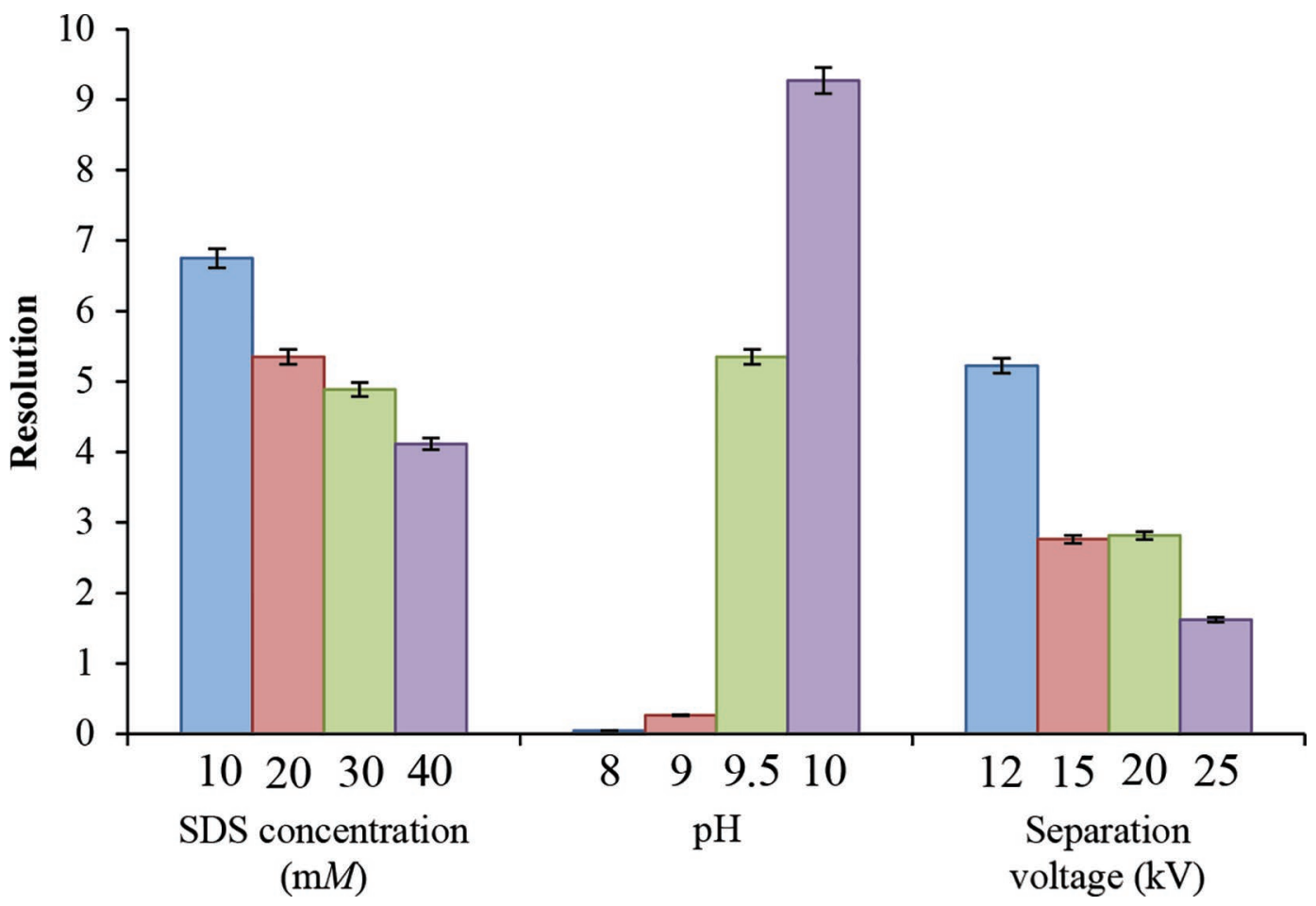

Figure 2. Optimization of separation conditions. Electropherograms of genistein and biochanin A standards $(0.5 \mathrm{mg} / \mathrm{mL}$ each). Background electrolyte composition: $30 \mathrm{~m} M$ sodium borate, SDS (10-40 mM) containing 5\% (vol/vol) ethanol (pH 8-10), separation voltage (12-25 $\mathrm{kV}$ ); detection: $254 \mathrm{~nm}$; injection: $3.5 \mathrm{kPa}$ for $3 \mathrm{~s}$; capillary: $75-\mu \mathrm{m}$ diameter; total length: $64.5 \mathrm{~cm}$; effective length: $56 \mathrm{~cm}$. Error bars represent deviation of 3 repeated measurements. 
migration times were significantly higher. Therefore, $\mathrm{pH} 9.5$ was chosen to speed up the separation time. We found that genistein has a shorter migration time compared with biochanin A. This is probably caused by the methyl group in the molecular structure of biochanin A. This group increases the hydrophobicity of these molecules and increases the affinity to the pseudostationary phase, which leads to the longer migration time.

\section{Template Removal Optimization}

The template removal step is extremely important in the process of MIP preparation. In this step, the bound template is washed out from the polymeric structure, and material with recognition sites selective for imprinted molecules is obtained (Batlokwa et al., 2011).

During the template removal phase, undesired damage of analyte binding sites can happen. Recognition sites in the polymeric layer can also change their structure due to the solvent composition, resulting in polymer swelling or other unfavorable effects (Ansell, 2015). So, the choice of a suitable solvent and appropriate removal approach are essential. For template removal, we tested 5 solvents: $0.5 \mathrm{M} \mathrm{NaCl}, 90 \%$ methanol, $80 \%$ ethanol, $20 \mathrm{~m} M$ Tris buffer ( $\mathrm{pH} 10)$, and a mixture of $1 \%$ SDS and $5 \%$ acetic acid in a ratio of 1:1. From obtained data shown in Figure 3A, it can be concluded that the most appropriate solvent was $90 \%$ methanol. This solvent enabled us to remove more than $80 \%$ of bound template from the polymer layer with 1 washing. It is possible that methanol has the highest efficiency of all chosen solvents because used phytoestrogens are hydrophobic molecules and therefore are more soluble in hydrophobic solvents.

Subsequently, we carried out the experiment focused on optimizing the number of removal steps. Obtained data are shown in Figure 3B. The first bar represents the signal of genistein at the same concentration used for MIP preparation, and other bars show the signal of genistein present in the volume of $50 \mu \mathrm{L}$ of $90 \%$ methanol used for MIP or NIP layer washing. As shown, in the first washing step, $81.5 \%$ of used template is removed, and in the last washing aliquot, $2.4 \%$ of the original genistein amount is washed out and detected. Based on these results, it was determined that the MIP layer has to be washed 3 times to be applicable for subsequent use.

\section{Adsorption Experiments}

Binding Properties. To assess the binding properties of created MIP and NIP, we carried out an experi- ment in which different initial concentrations of genistein $(0.0075,0.015,0.03,0.06,0.125,0.25$, and $0.5 \mathrm{mg} /$ $\mathrm{mL}$ ) in the sample (standard solution) were used. Obtained data (Figure 4A) enabled us to compare the binding capacity of MIP against NIP. We found that the amount of genistein in MIP rapidly increased with the increasing concentration and reached adsorption saturation at $0.3 \mathrm{mg} / \mathrm{mL}$. The method based on the MIP layer provided a possibility of genistein detection within the linear range $\left(\mathrm{R}^{2}=0.98\right)$ of 2.2 to $27 \mu \mathrm{M}$, demonstrating a detection limit of $0.59 \mu M$ and a limit of quantification of $22 \mu M$.

To evaluate the ability of MIP to bind the analyte, imprinting factor (IF) was used. The IF indicates the ratio of the distribution ratio for a specific analyte, under a particular set of conditions, on the imprinted polymer, to the distribution ratio for the same analyte, under identical conditions, on the NIP. We calculated the IF according to Equation 1:

$$
\mathrm{IF}=\frac{\mathrm{D}_{\mathrm{MIP}}}{\mathrm{D}_{\mathrm{NIP}}}=\frac{n_{\text {bound MIP }}}{\mathrm{M}_{\mathrm{MIP}}} \times \frac{V}{n_{\text {free }}},
$$

where $\mathrm{D}_{\mathrm{MIP}}$ and $\mathrm{D}_{\mathrm{NIP}}$ are distribution ratios, $\mathrm{M}_{\mathrm{MIP}}$ is the mass of MIP polymer, $V$ is the volume of solution in which the material is incubated, $n_{\text {bound }}$ is amount of bound analyte and $n_{\text {free }}$ is the amount of free analyte in solution.

The value of IF should be greater than 1 . The higher the difference between the imprinted and nonimprinted polymer, the greater the value of IF (Ansell, 2015; Mattiasson and Ye, 2015). The values of IF of genistein and biochanin A are shown in Table 1. It is shown that the value of IF in case of genistein is 8.81 , which mean that the difference between MIP and NIP is sufficient and prepared MIP can be used for isolation of genistein.

Adsorption Kinetics. Further, we investigated adsorption kinetics of prepared MIP and NIP by interaction of MIP and NIP with genistein for different time intervals (5-35 min). Figure 4B shows the curve of adsorption kinetics of $0.5 \mathrm{mg} / \mathrm{mL}$ genistein on prepared MIP and NIP. At the beginning, the binding capacity of genistein increased rapidly, which was caused by a large number of empty cavities available to target molecules. Gradually, the adsorption rate slowed and reached the maximum after $25 \mathrm{~min}$. After $30 \mathrm{~min}$, most of the binding sites were occupied by molecules of genistein. The cavities for selective binding of target molecules are not present on the surface of NIP. Therefore, lower values are reported for interaction with the target molecule. In the case of NIP, only nonspecific adsorption occurred.

Moreover, the influence of MIP and NIP performance on polymerization time was investigated. Polymeriza- 
tion conditions such as dopamine concentration, $\mathrm{pH}$, presence of oxygen, and presence of compounds containing the amine group (e.g., Tris) have a great influence on polymeric layer formation (e.g., mechanism of polymerization, polymerization rate) and therefore on the properties of the polymeric layer (e.g., resulting layer thickness).

For this reason, the effect of duration of polymerization (which translates into layer thickness) on the performance of MIP and NIP was observed. The MIP
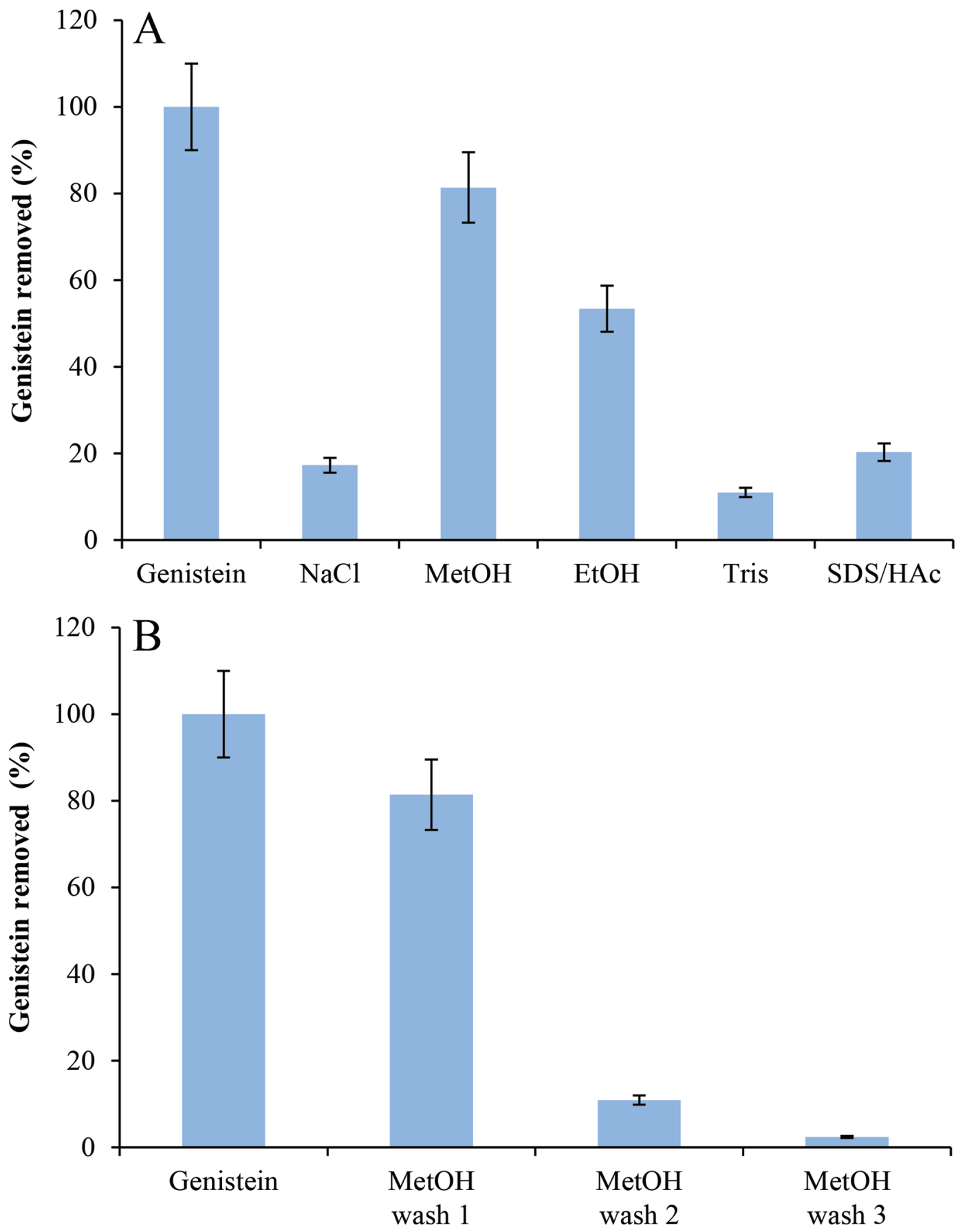

Figure 3. Optimization of template removal. (A) Percentage of the genistein removed from the molecularly imprinted polymer (MIP) surface. Genistein = capillary electrophoresis analysis of $0.5 \mathrm{mg} / \mathrm{mL}$ standard without MIP process; $\mathrm{NaCl}=0.5 \mathrm{M} \mathrm{NaCl} ; \mathrm{MetOH}=90 \% \mathrm{methanol}$; $\mathrm{EtOH}=80 \%$ ethanol; Tris $=20 \mathrm{~m} M$ Tris buffer $(\mathrm{pH} 10)$; SDS $/ \mathrm{HAc}=$ mixture of $1 \%$ SDS and $5 \%$ acetic acid in a ratio of 1:1. (B) Percentage of genistein removed by $50 \mu \mathrm{L}$ of $90 \%$ MetOH in 3 consecutive washes. Error bars represent deviation of 3 repeated measurements. 
extraction of genistein was measured using micellar electrokinetic chromatography after polymerization for several durations $(1.5,3,8,16,24$, and $72 \mathrm{~h})$. The obtained results are shown in Supplemental Figure S4
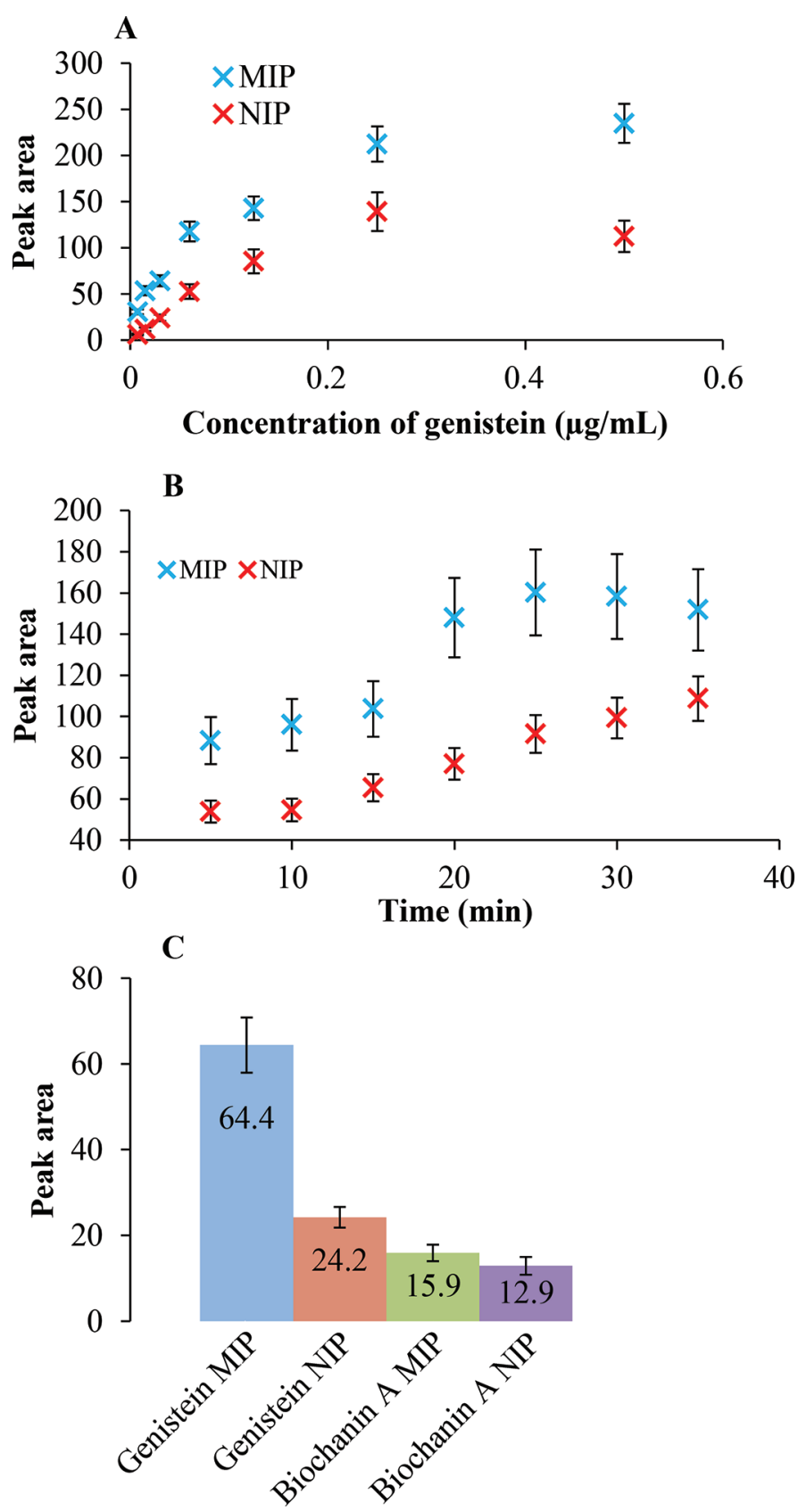

Figure 4. Binding properties and adsorption kinetics. (A) Saturation of the molecularly imprinted polymer (MIP) or nonimprinted polymer (NIP) layer prepared as described in the experimental section. Analysis of MIP/NIP layer with analytes of different concentrations. (B) Kinetics of adsorption investigation. Analysis of MIP/NIP layer with analytes after different times. (C) Selectivity investigation. Analysis of MIP/NIP layer with analytes different from the imprinted molecule. Error bars represent deviation of 3 repeated measurements. (https://doi.org/10.3168/jds.2019-17367). It is clear from the results that effectivity increased with increased time of polymerization and that optimum was reached after $24 \mathrm{~h}$. Moreover, after $72 \mathrm{~h}$ the nonspecific interaction increased significantly. Therefore, 24-h polymerization was carried out in all experiments.

\section{Selectivity of Prepared Polymeric Layers}

Moreover, we carried out the interaction of MIP and NIP with a competitor analyte to verify the selectivity. Biochanin A was chosen as the competitor. This molecule has a very similar structure to that of genistein but differs by the presence of the methoxy instead of hydroxyl group. The design of the experiment was as follows. To the MIP or NIP selective for genistein, biochanin A was added as a sample. The interaction was carried out for $25 \mathrm{~min}$ using the same procedure as described previously. Subsequently, we determined the amount of bound biochanin A and compared it with the amount of genistein isolated using the same process. The obtained data are shown in Figure 4C. It can be seen that MIP created to be selective for genistein bound biochanin A 4 times less than genistein.

To evaluate the selectivity, we calculated the factor of selectivity $\alpha$ according to Equation 2:

$$
\alpha=\mathrm{IF}_{\text {analyte MIP }} / \mathrm{IF}_{\text {competitor MIP }},
$$

where $\mathrm{IF}_{\text {analyte MIP }}$ and $\mathrm{IF}_{\text {competitor MIP }}$ are IF of studied analytes. The value of $\alpha$ should be greater than 1 , and high values of $\alpha$ for competitor provide evidence of selectivity. A value of selectivity factor of 6.94 confirms very good selectivity of prepared MIP.

\section{Isolation from Real Samples}

Complex matrixes as well as low concentrations complicate the determination of isoflavones. The comparison of concentrations detected using various methods is given in Table 2. Sample preparation is a crucial step; however, it is time consuming and laborious. Most common isolation techniques, such as liquid-liquid extrac-

Table 1. Factors of imprinting and selectivity for chosen phytoestrogens $^{1}$

\begin{tabular}{lccc}
\hline Analyte & $\begin{array}{c}\mathrm{D}_{\mathrm{MIP}} \\
\left(\mathrm{L} \cdot \mathrm{g}^{-1}\right)\end{array}$ & $\begin{array}{c}\mathrm{D}_{\mathrm{NIP}} \\
\left(\mathrm{L} \cdot \mathrm{g}^{-1}\right)\end{array}$ & $\begin{array}{c}\text { Imprinting } \\
\text { factor }\end{array}$ \\
\hline Genistein & 0.150 & 0.017 & 8.81 \\
Biochanin A & 0.006 & 0.005 & 1.27 \\
\hline
\end{tabular}

${ }^{1} \mathrm{D}_{\mathrm{MIP}}=$ distribution ratio of molecularly imprinted polymers (MIP); $\mathrm{D}_{\mathrm{NIP}}=$ distribution ratio of nonimprinted polymers (NIP). 
Table 2. Comparison of existing methods of genistein detection in bovine milk samples

\begin{tabular}{lccl}
\hline Method of genistein detection & $\begin{array}{c}\text { Limit of detection } \\
(\mathrm{ng} / \mathrm{mL})\end{array}$ & $\begin{array}{c}\text { Determined content } \\
\text { in milk }(\mathrm{ng} / \mathrm{mL})\end{array}$ & Reference \\
\hline $\begin{array}{l}\text { Liquid chromatography-tandem MS } \\
\text { HPLC with absorbance detection }\end{array}$ & 0.5 & $0.0-5.8$ & $\begin{array}{l}\text { Antignac et al. (2004) } \\
\text { Liquid chromatography-tandem MS }\end{array}$ \\
$\begin{array}{l}\text { HPLC diode array detector } \\
\text { Liquid chromatography-tandem MS }\end{array}$ & -5.8 & $4.0-29.0$ & $\begin{array}{l}\text { Antignac et al. (2003) } \\
\text { Hidalgo et al. (2004); Hoikkala et al. (2007) }\end{array}$ \\
\hline
\end{tabular}

tion and solid-phase extraction, are environmentally unfriendly and offer low selectivity and specificity. To overcome these obstacles, an MIP-based approach is beneficial (Chrzanowska et al., 2015b). The molecular imprinting materials for analysis of phytoestrogens have been investigated, and the topic has been extensively studied by Chrzanowska et al. (2015a, 2019).

In this work, we verified the applicability of the developed method by isolating genistein from real sample of bovine milk (Pragolaktos, Prague, Czech Republic). Dairy cow feed contains, among other ingredients, soybeans, legumes, and clover, which are rich in phytoestrogens. Sources in the literature confirm that phytoestrogens contained in feed can be transferred to milk (Krajčová et al., 2010; Bláhová et al., 2016). Therefore, in this work we tested the isolation of genistein from bovine milk samples. Figure 5 shows electropherograms of bovine milk samples. In general, crude bovine milk is a very complex matrix because of the presence of a range of proteins, sugars, and other molecules (blue trace). Despite the separation power of capillary electrophoresis, the analysis of such matrix may be difficult, especially when using relatively nonselective detection such as UV-visible absorbance detection. Even though this detection technique is the most universal, the low selectivity may cause certain difficulties. Therefore, the analyte isolation by MIP is beneficial (red trace).

\section{CONCLUSIONS}

We demonstrated that polydopamine-based MIP is an effective, eco-friendly approach to extracting phytoestrogens from complex matrices, including bovine milk. The approach presented here and the developed method are simple, selective, and compatible with the green chemistry philosophy. Finally, the combination with capillary electrophoresis, known as a separation method with extremely low consumption of chemicals, enabled us to create the presented bioanalytical method.

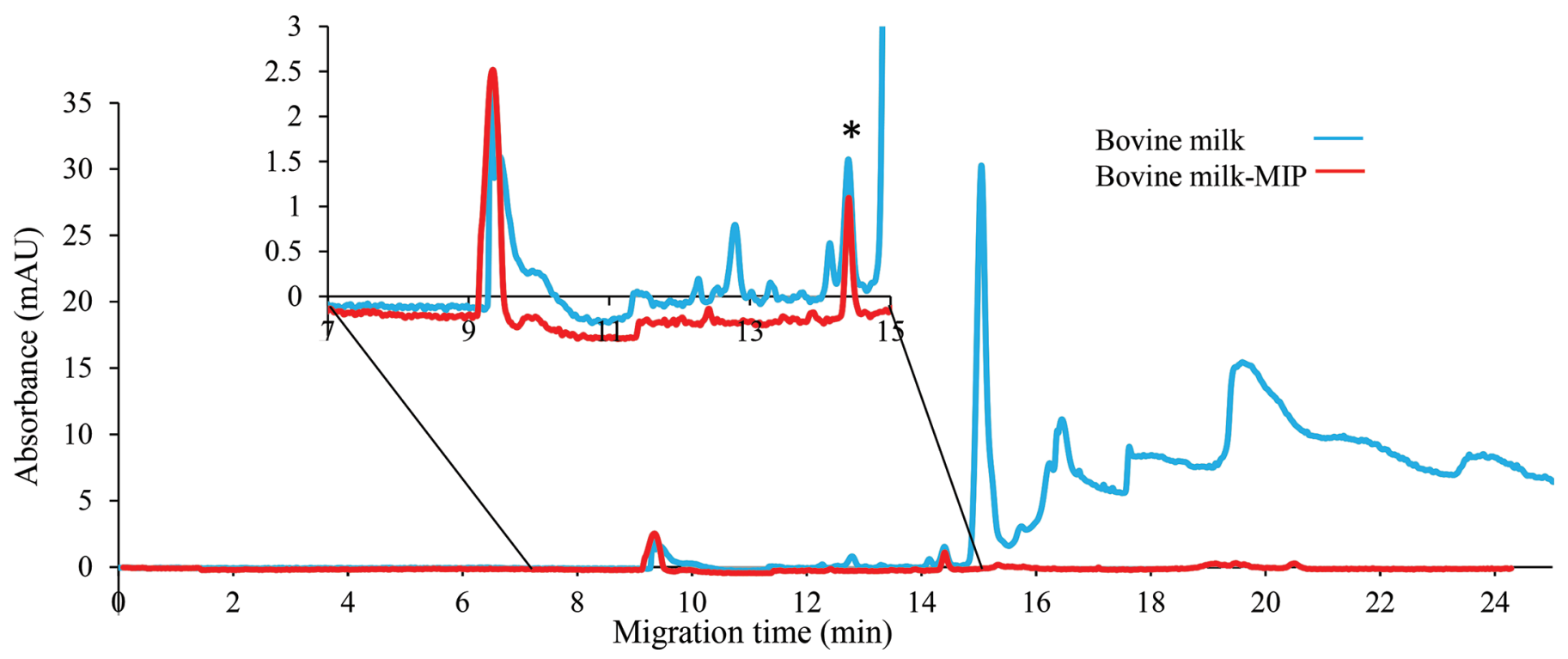

Figure 5. Electropherogram of crude bovine milk sample and the milk sample. Bovine milk = analyzed without any pretreatment. Bovine milk-MIP = bovine milk pretreated with molecularly imprinted polymer (MIP). Background electrolyte composition: $30 \mathrm{~m} M$ sodium borate, 20 $\mathrm{m} M$ SDS containing $5 \%$ (vol/vol) ethanol (pH 9.6). Detection: $254 \mathrm{~nm}$; injection: $3.5 \mathrm{kPa}$ for $3 \mathrm{~s}$; capillary: $75-\mu \mathrm{m}$ diameter; total length: 64.5 $\mathrm{cm}$; effective length: $56 \mathrm{~cm}$. mAU = milli-absorbance units. Genistein signal marked by asterisk. 


\section{ACKNOWLEDGMENTS}

This research was financially supported by the internal grant agency Mendelu IGA grant no. TP_4/2017. This research was carried out under the project CEITEC 2020 (LQ1601) with financial support from the Ministry of Education, Youth and Sports of the Czech Republic under the National Sustainability Programme II. We acknowledge Ceitec Nano Research Infrastructure supported by MEYS CR (LM2018110), the core facility of nanobiotechnology supported by MEYS CR (LM2018127), as well as the core facility of nanobiotechnology supported by MEYS CR (LM2015043). The authors declare no conflict of interest.

\section{REFERENCES}

Ansell, R. J. 2015. Characterization of the binding properties of molecularly imprinted polymers. Pages 51-93 in Molecularly Imprinted Polymers in Biotechnology. Vol. 150. B. Mattiasson and L. Ye, ed. Springer International Publishing AG, Cham, Switzerand.

Antignac, J. P., R. Cariou, B. Le Bizec, and F. Andre. 2004. New data regarding phytoestrogens content in bovine milk. Food Chem. 87:275-281. https://doi.org/10.1016/j.foodchem.2003.12.013.

Antignac, J. P., R. Cariou, B. Le Bizec, J. P. Cravedi, and F. Andre. 2003. Identification of phytoestrogens in bovine milk using liquid chromatography/electrospray tandem mass spectrometry. Rapid Commun. Mass Spectrom. 17:1256-1264. https://doi.org/10.1002/ rcm.1052.

Ball, V. 2018. Polydopamine films and particles with catalytic activity. Catal. Today 301:196-203. https://doi.org/10.1016/j.cattod.2017 .01 .031 .

Batlokwa, B. S., J. Mokgadi, T. Nyokong, and N. Torto. 2011. Optimal template removal from molecularly imprinted polymers by pressurized hot water extraction. Chromatographia 73:589-593. https: //doi.org/10.1007/s10337-010-1884-3.

Bennetau-Pelissero, C., K. Latonnelle, A. Sequeira, and V. Lamothe. 2000. Phytoestrogens, endocrine disrupters from food. Analusis 28:763-775. https://doi.org/10.1051/analusis:2000280763.

Bláhová, L., J. Kohoutek, T. Prochazkova, M. Prudikova, and L. Blaha. 2016. Phytoestrogens in milk: Overestimations caused by contamination of the hydrolytic enzyme used during sample extraction. J. Dairy Sci. 99:6973-6982. https://doi.org/10.3168/jds .2016-10926.

Cao, Y., A. M. Calafat, D. R. Doerge, D. M. Umbach, J. C. Bernbaum, N. C. Twaddle, X. Y. Ye, and W. J. Rogan. 2009. Isoflavones in urine, saliva, and blood of infants: Data from a pilot study on the estrogenic activity of soy formula. J. Expo. Sci. Environ. Epidemiol. 19:223-234. https://doi.org/10.1038/jes.2008.44.

Capeletti, L. B. and J. H. Zimnoch. 2016. Fourier transform infrared and raman characterization of silica-based materials. In Applications of Molecular Spectroscopy to Current Research in the Chemical and Biological Sciences. Mark Stauffer, ed. IntechOpen, London, UK.

Chen, L., X. Y. Wang, W. H. Lu, X. Q. Wu, and J. H. Li. 2016. Molecular imprinting: Perspectives and applications. Chem. Soc. Rev. 45:2137-2211. https://doi.org/10.1039/C6CS00061D.

Chrzanowska, A. M., M. Diaz-Alvarez, P. P. Wieczorek, A. Poliwoda, and A. Martin-Esteban. 2019. The application of the supported liquid membrane and molecularly imprinted polymers as solid acceptor phase for selective extraction of biochanin A from urine. J. Chromatogr. A 1599:9-16. https://doi.org/10.1016/j.chroma.2019 .04 .005 .

Chrzanowska, A. M., A. Poliwoda, and P. P. Wieczorek. 2015a. Characterization of particle morphology of biochanin A molecularly imprinted polymers and their properties as a potential sorbent for solid-phase extraction. Mater. Sci. Eng. C Mater. Biol. Appl. 49:793-798. https://doi.org/10.1016/j.msec.2015.01.069.

Chrzanowska, A. M., A. Poliwoda, and P. P. Wieczorek. 2015b. Surface molecularly imprinted silica for selective solid-phase extraction of biochanin A, daidzein and genistein from urine samples. J. Chromatogr. A 1392:1-9. https://doi.org/10.1016/j.chroma.2015 .03.015.

Dreyer, D. R., D. J. Miller, B. D. Freeman, D. R. Paul, and C. W. Bielawski. 2013. Perspectives on poly(dopamine). Chem. Sci. (Camb.) 4:3796-3802. https://doi.org/10.1039/c3sc51501j.

Franke, A. A., L. J. Custer, and Y. Tanaka. 1998. Isoflavones in human breast milk and other biological fluids. Am. J. Clin. Nutr. 68:1466S-1473S. https://doi.org/10.1093/ajcn/68.6.1466S.

Fu, S. W., G. F. Zeng, S. H. Zong, Z. Y. Zhang, B. Zou, Y. Fang, L. Lu, and D. Q. Xiao. 2014. Systematic review and meta-analysis of the bone protective effect of phytoestrogens on osteoporosis in ovariectomized rats. Nutr. Res. 34:467-477. https://doi.org/10 .1016/j.nutres.2014.05.003.

González Cañete, N., and S. Durán Agüero. 2014. Soya isoflavones and evidences on cardiovascular protection. Nutr. Hosp. 29:1271-1282.

Hidalgo, A., M. Rossi, C. Pompei, and E. Casiraghi. 2004. Uracil as an index of hygienic quality in egg products. Ital. J. Food Sci. 16:429-436.

Hoikkala, A., E. Mustonen, I. Saastamoinen, T. Jokela, J. Taponen, H. Saloniemi, and K. Wähälä. 2007. High levels of equol in organic skimmed Finnish cow milk. Mol. Nutr. Food Res. 51:782-786. https://doi.org/10.1002/mnfr.200600222.

Jiang, J., L. P. Zhu, L. J. Zhu, B. K. Zhu, and Y. Y. Xu. 2011. Surface characteristics of a self-polymerized dopamine coating deposited on hydrophobic polymer films. Langmuir 27:14180-14187. https:/ /doi.org/10.1021/la202877k.

King, R. A., M. M. Mano, and R. J. Head. 1998. Assessment of isoflavonoid concentrations in Australian bovine milk samples. J. Dairy Res. 65:479-489. https://doi.org/10.1017/S0022029998002891.

Krajčová, A., V. Schulzová, J. Lojza, L. Kř́žová, and J. Hajšlová. 2010. Phytoestrogens in bovine plasma and milk-LC-MS/MS analysis. Czech J. Food Sci. 28:264-274. https://doi.org/10.17221/ 138/2010-CJFS.

Li, J. P., G. Wei, and Y. Zhang. 2012. Molecularly imprinted polymers as recognition elements in sensors. Pages 35-56 in Molecularly Imprinted Sensors: Overview and Applications. S. Li, Y. Ge, S. A. Piletsky, and J. Lunec, ed. Elsevier Science BV, Amsterdam, the Netherlands.

Mattiasson, B., and L. Ye, ed. 2015. Molecularly Imprinted Polymers in Biotechnology. Springer International Publishing AG, Cham, Switzerland.

Murkies, A. L., G. Wilcox, and S. R. Davis. 1998. Clinical review 92: Phytoestrogens. J. Clin. Endocrinol. Metab. 83:297-303.

Patisaul, H. B., and W. Jefferson. 2010. The pros and cons of phytoestrogens. Front. Neuroendocrinol. 31:400-419. https://doi.org/ 10.1016/j.yfrne.2010.03.003.

Perrot, D., C. Croutxe-Barghorn, and X. Allonas. 2016. Towards mussel-like on-demand coatings: Light-triggered polymerization of dopamine through a photoinduced $\mathrm{pH}$ jump. Polym. Chem. 7:2635-2638. https://doi.org/10.1039/C6PY00356G.

Sekine, R., E. G. Robertson, and D. McNaughton. 2011. Raman, infrared and computational analysis of genistein and its methoxy derivatives. Vib. Spectrosc. 57:306-314. https://doi.org/10.1016/j .vibspec.2011.09.005.

Socas-Rodríguez, B., J. Hernández-Borges, A. V. Herrera-Herrera, and M. A. Rodríguez-Delgado. 2018. Multiresidue analysis of oestrogenic compounds in cow, goat, sheep and human milk using core-shell polydopamine coated magnetic nanoparticles as extraction sorbent in micro-dispersive solid-phase extraction followed by ultra-high-performance liquid chromatography tandem mass spectrometry. Anal. Bioanal. Chem. 410:2031-2042. https://doi.org/10 $.1007 / \mathrm{s} 00216-018-0882-4$.

Terabe, S. 2009. Capillary separation: Micellar electrokinetic chromatography. Annu. Rev. Anal. Chem. 2:99-120. 
Thakur, A., S. Ranote, D. Kumar, K. Bhardwaj, R. Gupta, and G. Chauhan. 2018. Synthesis of a PEGylated dopamine ester with enhanced antibacterial and antifungal activity. ACS Omega 3:79257933. https://doi.org/10.1021/acsomega.8b01099.

Tham, D. M., C. D. Gardner, and W. L. Haskell. 1998. Clinical review 97: Potential health benefits of dietary phytoestrogens: A review of the clinical, epidemiological, and mechanistic evidence. J. Clin. Endocrinol. Metab. 83:2223-2235.

Wang, C. C., J. K. Prasain, and S. Barnes. 2002. Review of the methods used in the determination of phytoestrogens. J. Chromatogr. B Analyt. Technol. Biomed. Life Sci. 777:3-28. https://doi.org/10 .1016/S1570-0232(02)00341-0.

Xiao, C. W. 2008. Health effects of soy protein and isoflavones in humans. J. Nutr. 138:1244S-1249S. https://doi.org/10.1093/jn/138 .6.1244S.
Zhao, Y., Y. H. Tang, J. He, Y. Xu, R. X. Gao, J. J. Zhang, T. Chong, L. Wang, and X. S. Tang. 2018. Surface imprinted polymers based on amino-hyperbranched magnetic nanoparticles for selective extraction and detection of chlorogenic acid in Honeysuckle tea. Talanta 181:271-277. https://doi.org/10.1016/j.talanta.2018.01.037.

Zheng, W., Q. Dai, L. J. Custer, X. O. Shu, W. Q. Wen, F. Jin, and A. A. Franke. 1999. Urinary excretion of isoflavonoids and the risk of breast cancer. Cancer Epidemiol. Biomarkers Prev. 8:35-40.

Zhou, W. H., C. H. Lu, X. C. Guo, F. R. Chen, H. H. Yang, and X. R. Wang. 2010. Mussel-inspired molecularly imprinted polymer coating superparamagnetic nanoparticles for protein recognition. J. Mater. Chem. 20:880-883. https://doi.org/10.1039/B916619J. 